\title{
Optimization of parameters of neuro-fuzzy model
}

\author{
Lyalya Bakievna Khuzyatova, Lenar Ajratovich Galiullin \\ Kazan Federal University Naberezhnye Chelny Institute, Russia
}

\section{Article Info}

Article history:

Received Jun 26, 2019

Revised Aug 12, 2019

Accepted Aug 26, 2019

\section{Keywords:}

Fuzzy

Logic

Network

Programming

System

\begin{abstract}
The questions and problems of the formation of knowledge bases of intelligent man-machine decision support systems are considered. The neuron-fuzzy model used in the work is described. The need for increasing the efficiency of the neuron-fuzzy model in the formation of knowledge bases is being updated. The task is to develop methods and algorithms for presetting and optimizing the parameters of a fuzzy neural network. To solve difficult formalized tasks, it is necessary to develop decision support systems-expert systems based on a knowledge base. ES developers are constantly faced with the problems of "extraction" and formalization of knowledge, as well as the search for new ways to obtain it. To do this, use the extraction, acquisition and formation of knowledge. Currently, the formation of knowledge bases is relevant for the creation of hybrid technologies - fuzzy neural networks that combine the advantages of neural network models and fuzzy systems. The analysis of the efficiency of the fuzzy neural network carried out in the work showed that the quality of training of the NN largely depends on the choice of the number of fuzzy granules for input drugs. In addition, to use fuzzy information formalized by the mathematical apparatus of fuzzy logic, procedures are required for selecting optimal forms and presetting the parameters of the corresponding membership functions (MF).
\end{abstract}

Copyright $\odot 2020$ Institute of Advanced Engineering and Science. All rights reserved.

\section{Corresponding Author:}

Lenar Ajratovich Galiullin,

Kazan Federal University Naberezhnye Chelny Institute,

Russia.

Email: galilenar@yandex.ru

\section{INTRODUCTION}

Modern information systems for analyzing large amounts of information or managing complex processes cannot be imagined without elements of artificial intelligence [1-3]. Data mining methods allow building effective models of diagnostics, forecasting, decision making in many subject areas of human activity [4-10].

Such models are used in a wide class of intelligent information systems, especially in expert systems (ES), the main element of which is the knowledge base - a model represented by many systematized rules that describe patterns in the subject area under consideration. Therefore, the design of knowledge bases is an important task in the development of expert systems [11,12].

The analysis of the capabilities of the neuro-fuzzy knowledge base formation model showed that the quality of training of a fuzzy neural network (NN) largely depends on the choice of the number of fuzzy granules for input linguistic variables (LV) [13-17]. In addition, to use fuzzy information formalized by the mathematical apparatus of fuzzy logic, procedures are needed for selecting optimal forms and initializing the parameters of the corresponding membership functions. For these reasons, to improve the accuracy of approximation of experimental data by fuzzy production rules, it is necessary to automatically select the optimal number of fuzzy granules of input linguistic variables of a fuzzy neural network and the corresponding forms and parameters of their membership functions. 


\section{METHODS}

Often, experts decide the problem of choosing the number of fuzzy gradations. For purely psychological reasons, they choose an odd number of values of a linguistic variable, for example, 3, 5, 7. Moreover, this choice is subjective and does not always reflect the real picture [18-20].

When using automatic methods, the choice of the number of values of a linguistic variable is based on the criterion of its optimality [21]. For this, methods of cluster data analysis are often used. To date, a large number of different algorithms have been developed and used to solve the clustering problem. The assumptions of traditional clustering algorithms determine the following factors that do not allow their full application in the developed methodology:

a) A priori assumptions about the properties of clusters, the principles of combining objects, or setting the number of clusters are unacceptable;

b) It is unacceptable to construct an algorithm only on the relation of points to the centers of clusters, and not on the basis of the relative positions of the points.

c) The absence of an understandable linguistic interpretation of partitions is unacceptable.

In this regard, to effectively solve the problem of clustering the values of the input parameters of a fuzzy neural network, the development of a special algorithm that takes into account the relationship between individual data points without being tied to the center of the cluster is relevant.

The developed clustering algorithm is based on a fuzzy relationship apparatus using the concepts of $\alpha$-tolerance and $\alpha$-quasi-equivalence relations, which, respectively, make sense of pairwise comparison of data samples relative to a given sample and intergroup data comparison. To build a family of $\alpha$-quasiequivalence relations, a sequence of data comparison methods is used, each of which is based on the previous one and is more suitable for directly solving the fuzzy clustering problem [22].

a) Comparison by distance between data samples - pairwise comparison of data samples.

b) Comparison using normal measures of similarity - one by one comparison of all data samples with each of the samples. The result is fuzzy sets of data samples that are close to each of the data samples.

c) Comparison using relative similarity measures - pairwise comparison of data samples relative to a given sample. The result is the degree to which each pair of data samples belongs to the corresponding similarity relationship.

d) Comparison of data samples using the ratio of $\alpha$-tolerance on many data samples - the similarity of any two data samples relative to all other samples. The result is the degree to which each pair of data samples belongs to the $\alpha$-tolerance ratio.

e) Comparison using the ratio of $\alpha$-quasi-equivalence and the scale of the ratio of $\alpha$-quasi-equivalence is an intergroup similarity of data. The result is the degree to which each pair of data samples belongs to the ratio of $\alpha$-quasi-equivalence.

The clustering method in the algorithm is the use of a family of equivalence relations, each of which is obtained by switching from the a-quasi-equivalence relation to the equivalence relation in the classical sense using the corresponding level of a-quasi-equivalence relation from the scale of a-quasi-equivalence relation. Those data samples that, in accordance with the a-quasi-equivalence relation, have similarities exceeding the indicated level are equivalent, the rest are nonequivalent.

Cluster partitioning is fuzzy - corresponding to the presence of a fuzzy data relationship. The number of partitions is finite and is determined by the power (the number of ratio levels) of the ratio scale of a-quasi-equivalence. Each concrete partition by clusters corresponds to a partition of the set of data samples into equivalence classes at a certain level of a-quasi-equivalence.

Let the values of the input parameters of the neural network xi are given on a nonempty set $\mathrm{X}$ and the clustered object represents only one feature. We introduce the basic concepts and definitions. A normal measure of similarity in distance $x_{q}$ with $x_{i}$ is a measure that reaches its boundary values on the set $\mathrm{X}$ with the membership function, defined as follows.

\section{RESULTS AND DISCUSSION}

When training a fuzzy neural network, a study was conducted of the effectiveness of the technique. The quality of $\mathrm{NN}$ training in changing its output error was evaluated for various approaches to choosing the number of gradations of input drugs and the forms of their membership functions. Using a technique based on the developed algorithms, the quality of NN training was not inferior, and in many cases, superior to the quality of network training, in which the choice of the number of gradations of input neurons and forms of $\mathrm{AF}$ was determined subjectively by an expert. The considered example showed that when using the proposed methodology, the quality of training of the NN significantly improved. This indicates the practical feasibility and advisability of using this technique. Consider the problem of choosing the optimal forms of membership functions of the input LP values. The membership functions of a fuzzy set are traditionally built on expert 
information. There are a significant number of such methods that can be divided into two groups: direct and indirect. The simulation took place in the SimInTech system. [23-28]

Examples of direct methods are the direct assignment of MF to a table, schedule, or formula. In indirect methods, the values of the phase transitions are chosen in such a way as to satisfy predefined conditions. Expert information is only the initial information for constructing a function.

The disadvantage of both groups of methods is a large share of subjectivity. A different approach to the construction of phase transitions is based on the parametric identification of fuzzy models based on experimental "input-output" data. Using this approach removes the subjectivity of constructing functions, but instead requires a training sample with representative examples of "inputs - output." In addition, phase transitions of identical fuzzy sets in meaning are obtained differently as a result of identification of various "input-output" dependencies.

\section{SUMMARY}

Currently, in fuzzy modeling systems, the most common among experts are triangular, trapezoidal, and Gaussian membership functions. In the proposed approach, for modeling fuzzy constraints, in addition to the three indicated, double and double Gaussian phase transitions are also used as generalizations of the trapezoidal and triangular functions, respectively.

We assume that the observed observations and the processes under study obey a certain law described by the mathematical model, and deviations from it are random. In this case, the least squares method is best for estimating the accuracy of the approximation. Using this method and evaluating the residual variance, we determine the shape and initialize the parameters of the phase transition that most accurately describes the initial fuzzy set.

\section{CONCLUSIONS}

The analysis of the effectiveness of data mining methods and strategies for obtaining knowledge for expert systems has been carried out in order to justify the relevance of developing new mathematical methods and algorithms for the automated generation of ES knowledge bases, as well as the need to increase the efficiency of one of such methods, the neuron-fuzzy model.

\section{ACKNOWLEDGEMENTS}

The work is performed according to the Russian Government Program of Competitive Growth of Kazan Federal University

\section{REFERENCES}

[1] L.A. Galiullin, R.A. Valiev, "Modeling of internal combustion engines test conditions based on neural network," (2016) International Journal of Pharmacy and Technology, 8 (3), pp. 14902-14910.

[2] E.V. Zubkov, A.A. Novikov, "Regulation of the Crankshaft Speed of a Diesel Engine with a Common Rail Fuel System," Russian Engineering Research, vol. 32, no. 7-8, pp. 523-525, 2012.

[3] D. Wei, "Design of Web based expert system of electronic control engine fault diagnosis," BMEI 2011 Proceedings 2011 International Conference on Business Management and Electronic Information, 1, art. no. 5916978, pp. 482 - 485, 2011.

[4] E.V. Zubkov, A.A. Makushin, A.A. Novikov, A.A. Gafijatullin, "Imitating modeling of testing of the diesel with Common Rail fuel supply system," Assembling in Mechanical Engineering and Instrument-Making, no. 8, pp. 29-31, 2011

[5] L.A. Galiullin, R.A. Valiev, "An automated diagnostic system for ICE," (2018) Journal of Advanced Research in Dynamical and Control Systems, 10 (10 Special Issue), pp. 1767-1772.

[6] E.V. Zubkov, L.A. Galiullin, "A hybrid neural network for modeling of the modes of tests of internal combustion engines," no. 1, pp. 245-250, 2011.

[7] R. Ahmed, M. El Sayed, S.A. Gadsden, J. Tjong, S. Habibi, "Automotive internal-combustion-engine fault detection and classification using artificial neural network techniques," IEEE Transactions on Vehicular Technology, 64 (1), № 6803044, pp. 21-33, 2015.

[8] E.V. Zubkov, D.I. Mochalov, A.H. Hajrullin, "Imitating modeling of technological processes of the automated tests of diesels at dynamic loads at machine-building enterprise," Scientific and Technical Volga region Bulletin, no. 1, pp. 274-277, 2013.

[9] L.A. Galiullin, R.A. Valiev, "Method for neuro-fuzzy inference system learning for ICE tests," (2018) Journal of Advanced Research in Dynamical and Control Systems, 10 (10 Special Issue), pp. 1773-1779. 
[10] A.N. Ilyukhin, E.V. Zubkov, "Modernized algorithm of neural network initial weighting factors during the diagnosis of diesel engine faults," International Journal of Applied Engineering Research, 10 (24), pp. 44848-44854, 2015.

[11] J. Chen, R. Randall, N. Feng, B. Peeters, H. Van Der Auweraer, “Automated diagnosis system for mechanical faults in IC engines," 10th International Conference on Condition Monitoring and Machinery Failure Prevention Technologies, CM 2013 and MFPT 2013, 2, pp. 892-903, 2013.

[12] A.A. Makushin, E.V. Zubkov, A.N. Ilyukhin, "Fuzzy Logic in the Modeling of Tests for Internal Combustion Engines," Assembling in Mechanical Engineering and Instrument-Making, no. 12, pp. 39-44, 2009.

[13] J. Chen, R. Randall, B. Peeters, W. Desmet, H. Van Der Auweraer, "Neural network based diagnosis of mechanical faults in IC engines," Institution of Mechanical Engineers - 10th International Conference on Vibrations in Rotating Machinery, pp. 679-690., 2012.

[14] Baum F.I., "SimInTech software for programming control system devices / F.I. Baum, O.S. Kozlov, I.A. Parshikov, V.N. Petukhov, K.A. Timofeev, A.M. Shchekaturov," Atomic energy, 2012.V.113. Issue 6 P.354-357.

[15] J.-D. Wu, C.-K. Huang, Y.-W. Chang, Y.-J. Shiao, "Fault diagnosis for internal combustion engines using intake manifold pressure and artificial neural network," Expert Systems with Applications, 37 (2), pp. 949-958, 2010.

[16] B.A. Kartashov, E.A. Shabaev, O.S. Kozlov, A.M. Shchekaturov "Dynamic modeling environment for technical systems SimInTech," - Moscow: DMK Press, 2017. - pp. 423.

[17] D. Danfeng, M. Yan, G. Xiurong, “Application of PNN to fault diagnosis of IC engine," 2009 2nd International Conference on Intelligent Computing Technology and Automation, ICICTA 2009, 2, № 5287738, pp. 495-498, 2009.

[18] Lenar A. Galiullin; Rustam A. Valiev, "Internal combustion engine fault simulation method," IIOAB OURNAL, №9, pp. 91-96, 2018.

[19] E.V. Zubkov, A.A. Makushin, A.N. Ilyukhin, "Rules, Functions, and Systems for the Formation of Models of the Test Conditions for Truck and Tractor Combustion Engines," Traktors Selkhozmash, no. 5, pp. 17-20, 2009.

[20] Lenar A. Galiullin; Rustam A. Valiev, Khairullin A. Haliullovich, "Method for modeling the parameters of the internal combustion engine,” IIOAB JOURNAL, №9, pp. 83-90, 2018.

[21] R.A. Valiev, A.Kh. Khairullin, V.G. Shibakov, "Automated Design Systems for Manufacturing Processes," Russian Engineering Research, 35(9). pp. 662 - 665, 2015.

[22] Lenar A. Galiullin; Rustam A. Valiev, "Control vector for ice automated test and diagnostic system," Dilemas Contemporaneos-Educacion Politica Y Valores, №6 SI, article №95, 2018.

[23] Baum F.I. SimInTech software for programming control system devices / F.I. Baum, O.S. Kozlov, I.A. Parshikov, V.N. Petukhov, K.A. Timofeev, A.M. Shchekaturov // Atomic energy, 2012.V.113. Issue 6 P.354-357.

[24] B.A. Kartashov, E.A. Shabaev, O.S. Kozlov, A.M. Shchekaturov "Dynamic modeling environment for technical systems SimInTech," - Moscow: DMK Press, 2017. - pp. 423.

[25] Gutiérrez-Artacho, J., \& Olvera-Lobo, M. (2017). Web Localization of Spanish SMEs: The Case of Study in Chemical Sector. Journal of Information Systems Engineering \& Management, 2(3), 15. https://doi.org/10.20897/jisem.201715

[26] Sakthive, V., Kesaven, P. V., Martin William, J., \& Madan Kumar, S. K. (2019). Integrated platform and response system For healthcare using Alexa. International Journal of Communication and Computer Technologies, 7 (1), 14-22.

[27] Khodashenas, B. (2015). Nitrate reductase enzyme in Escherichia coli and its relationship with the synthesis of silver nanoparticles. UCT Journal of Research in Science, Engineering and Technology, 3(1), 26-32.

[28] Gadge, G., Sabale, V., Khade, A. \& Mahajan, U. (2019). Current Approaches on Gastro Retentive Drug Delivery System: An Overview. International Journal of Pharmacy Research \& Technology, 9 (2), 16-28. 